\title{
EL ESTATUTO NOÉTICO DEL PRIMUM COGNITUM. UNA APROXIMACIÓN DESDE SANTO TOMÁS DE AQUINO
}

\author{
Luis Romera Oñate \\ Ateneo Romano Santa Croce. Roma
}

\section{LA DIVERSIDAD OPERATIVA DE LA RAZÓN}

El tema que nos ocupa en estas sesiones se centra en la reflexión de los límites de la razón. Cuestionar dichos límites y -en paticular-estudiar las formas de conocimiento que superan a la razón supone, primero, dilucidar qué niveles o modos de racionalidad son, en cada caso, superados, y en qué medida lo son.

Es sabido que Aristóteles en el libro sexto de la Etica a Nicómaco sistematiza las diversas operatividades de la razón, distinguiendo el conocimiento teórico del práctico; en el primero, a su vez, distingue entre el modo de racionalidad propio del conocimiento de los principios, de las operaciones intelectuales que se ejercen en la ciencia, así como de las que se utilizan en la sabiduría. Dentro de la razón práctica deslinda el campo de la prudencia de aquel de la técnica. No confundir los actos intelectuales que se llevan a cabo en estas diversas vías de la razón es necesario para evitar confusiones y simplificaciones que pueden provocar una cierta desorientación.

La distinción aristotélica es plenamemnte asumida por Tomás de Aquino. La presente nota pretende determinar el estatuto noético particular que corresponde al primum cognitum.

Remitiéndose a Avicena, el doctor Angélico establece en numerosas ocasiones que lo primero que conoce la inteligencia, como lo más conocido y en lo que se resuelve todo conocimiento, es el ente. ${ }^{1}$ En ente como primer conocimiento ha sido objeto de frecuente debate entre los interprétes del pensador italiano. ${ }^{2}$

1 In Boeth. de Trinitate, q. 1, a. 3, ad 9; In I Sent., d. 25, q. 1, a. 4

2 Alcorta, J.L., Vacilaciones tomistas sobre el ser primum cognitum en «Folia Humanistica», 19, 1981, 187-195. R. T. Caldera, La primera captación intelectual . Torino-Caracas, 1988. 


\section{EN ENS COMO PRIMUM COGNITUM}

Para intentar determinar su naturaleza gnoseológica es preciso no perder de vista las peculiaridades de lo primero conocido.

Su primacía se establece no solamente a nivel psicológico-analítico, según la cual en la base de toda otra noción está presente analíticamente la de ente, ${ }^{3}$ sino que consiste también en un primero resolutivo-noético. En efecto, para Santo Tomás la inteligencia queda constituída por la captación del ente, es más, todo acto intelectivo se inicia con el ens y se resuelve en el ens. ${ }^{4}$ De ahí que sea el primer «objeto» del conocimiento y que configure toda la operatividad intelectual, entendiendo todo lo que entiende sub ratione entis.

El primado noético del ens implica -dicho telegráficamente- que todo conocimiento se hace por adición al ente, ${ }^{5}$ que el ente comprende toda especie y diferencia, ${ }^{6}$ en él se incluye todo conocimiento como en un principio, ${ }^{7}$ al primum cognitum no se le puede oponer nada a modo de privación o contrtariedad, sino simplemente por negación radical. ${ }^{8}$

De todo lo anterior se deduce que el primum cognitum no es un objeto captado en una primera operación intelectual para luego quedar almacenado en la conciencia, por el contrario, su primado se «ejerce» -está presente- en todo acto de conocimiento intelectual.

Siguiendo a Aristóteles, Tomás de Aquino excluye una consideración del mismo por línea de generalidades más universales e indeterminadas propias de los géneros: la dialéctica de género-especie-diferencia no se puede aplicar al caso del primum cognitum, el cual se comporta como un principio. ${ }^{9}$ El estatuto noético que le corresponde es, entonces, el de los principios. Intentemos precisarlo.

Santo Tomás, siguiendo la dicotomía griega nous-dianoia distingue en el intelecto humano un doble modo de operar. Uno discursivo, donde para llegar al conocimiento de las conclusiones, se parte de algo ya conocido y, razonando, alcanzamos su conocimiento. Otro no discursivo, sino inmediato, donde llegamos al conocimiento sin necesidad de razonar. $\mathrm{Al}$ primero llama ratio, al segundo intellectus. ${ }^{10}$ El intellectus y la ratio no son dos facultades distintas, sino actos diversos de la misma potencia. ${ }^{11}$ Estos dos actos intelectuales se comparan como el movimiento al reposo, el adquirir al tener: como lo imperfecto a lo perfecto. ${ }^{12}$ Todo discurso de la razón empieza en el intellectus y en él termina, ${ }^{13}$ de tal modo que la razón se

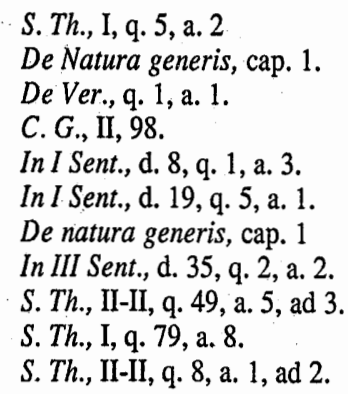


refiere al intelecto como a su principio y término, porque todo proceso de raciocinio se inicia con uno intelectivo y acaba también en uno intelectivo. ${ }^{14}$

Al intellectus le corresponde el conocimiento de los primeros principios; los cuales son tenidos a modo de hábito: el hábito de los primeros principios. ${ }^{15}$ Todo proceso de búsqueda 0 de descubrimiento procede a partir de unas primeras intelecciones, inteligidas de modo simple -los primeros principios- a las cuales vuelve la inteligencia para, en la vía del juició y resolviendo, examinar a su luz lo hallado. ${ }^{16} \mathrm{El}$ intelecto, partiendo del conocimiento inmediato de los primeros principios-nous-, llega, por el raciocinio -dianoia-, a las conclusiones. ${ }^{17} \mathrm{Es}$ decir, en la inteligencia tenemos unos conocimientos per se noti, que son primeros y se perciben statim por el intellectus (hábito de los primeros principios) por medio de los cuales llegamos al conocimiento de todo lo demás. ${ }^{18}$

Pero en Santo Tomás, además de estos principios que se captan inmediatamente, evidentes para todos y por los que la razón procede par al conocimiento de cualquier cosa, también hay quaedam conceptiones omnibus notae ut entis, unius, boni, de las cuales parte el entendimiento para el conocimiento de las esencias. ${ }^{19}$

En el De Veritate ${ }^{20}$ señala también que el entendimiento tiene unas primeras concepciones, que inmediatamente captamos por la luz del intelecto agente a partir de lo sensible, que son complejas -como los primeros principios- o incomplejas - como ente y uno-y de las cuales se sigue todo conocimiento. Es decir, distinguiendo al ens de los primeros principios, afirma no obstante que el lugar que ocupan en la inteligencia es semejante, estableciendo un paralelismo entre lo primero que es el ens y los primeros principios, indicando que todo se incluye en el ente como en un principio. ${ }^{21}$

14 De Ver., q. 15, a. 1.

15 In III Sent., d. 35, q. 2, a. 2.

16 S. Th., I, q. 79, a. 8.

17 C. G., I, 76; In III de An., 1. 14, n. 812.

18 S. Th., I-II, q. 57, a. 2. No obstante recuérdese que al tratar de las proposiciones evientes como proposiciones per se notae, se debe distinguir entre enunciados per se notum quoad nos y per se notum quoad se. Al respecto, M. Dummett (Frege, Philosophy of language, Duckword, London, 1973) trae a colación la distinción entre necesidad real necesidad epistemológica; por ejemplo, la proposición «Dios existe» es necesidad ontológica pero no de necesidad epistemológica (pp. 117-120). La filosofía es el proceso que nos lleva de lo evidente quoad nos (princpios epistemológicos) a lo que es evidente quoad se (princpios ontológicos).

19 Quodlibeto, VII, q. 2, a. 2: «Insunt nobis naturaliter quaedam principia complexa omnibus nota, ex quibus ratio procedit ad cognoscendum in actum conclusiones quae praedictis principiis potentialiter continentur (...). Et similiter in intellectu insunt nobis etiam naturaliter quaedam conceptiones omnibus notae, ut entis, unius, boni, et huiusmodi, a quibus eodem modo procedit intellectus ad cognoscendum quidditatem uniuscuiusque rei, per quem procedit a principiis per se notis ad cognoscendas conclusiones».

20 De Ver., q. 11, a. 1: «Praexistunt in nobis quaedam scientiarum semina, scilicet primae conceptiones intellectus, quae statim, lumine intellectus agentis cognoscuntur per species a sensibilibus abstractas, sive sint complexa, ut dignitates, sive incomplexa, sicut ratio entis et unius, et huiusmodi, quae statim intellectus apprehendit. Ex istis autem principiis universalibus omnia principia sequuntur sicut ex quidam rationes seminalibus».

21 In I Sent., d. 8, q. 1, a. 3. 
Por tanto, podemos concluir que la noción de ens se sitúa en el intellectus, de modo análogo a los primeros principios; lo cual significa que se capta statim, permanece en forma de hábito y es como un principio sobre el que se apoya el resto de conocimientos.

\section{LA DIMENSIÓN CONCOMITANTE DEL CONOCIMIENTO INTELECTUAL}

Ya hemos hecho notar cómo el primum cognitum es un primero en todo acto intelectivo, se vuelve a captar en todo conocimiento intelectual de la realidad. También hay otros conocimientos que aparecen en todo acto intelectivo o al menos en algunas modalidades de nuestro conocer. Detenernos en el análisis de estos elementos puede ofrecernos una vía de investigación.

El primero de ellos es el conocimiento de la propia subjetividad. Aristóteles había advertido que en el acto intelectual, no solamente conocemos aquello sobre lo que de un modo directo o temático versa dicho acto de intelección, sino que también al entender nos percatamos de que pensamos, y darse cuenta de que se piensa es percibir que somos..$^{22}$ Como escribe Millán Puelles, en toda vivencia cognoscitiva originaria de la conciencia se da una co-presencia, en planos distintos, de lo mundano y de la misma subjetividad. ${ }^{23}$

También Fabro se detiene en este elemento del acto cognoscitivo: junto a la percepción del objeto (sea el que sea), el acto cognoscitivo intelectual incluye en él a la misma subjetividad en la medida en que no sólo conozco, sino que también conozco que conozco. ${ }^{24}$ Este ser consciente de que conozco que acompaña al acto intelectual, es una conciencia inmediata y concomitante que aparece en cualquier acto psíquico consciente, llamado propiamente así por la presencia del yo a sí mismo como sujeto en acto respecto al conocer. ${ }^{25}$

La conciencia de la propia subjetividad en acto de entender (o de querer) presente en todo acto consciente debe distinguirse de la conciencia como reflexión posterior que hace la subjetevidad sobre sí misma, ${ }^{26}$ en donde el yo se transforma entonces en lo considerado directamente por el acto cognoscitivo. La conciencia que acompaña al acto intelectual es una cierta reflexión concomitante (in actu exercito), distinta de la conciencia que podemos llamar

22 Et. Nic., IX, 91170 a 29-33: «Cuando pensamos, (nos damos cuenta) de que estamos pensando, y percibir que sentimos o pensamos es percibir que somos.

23 Millán Puelles, A., La estructura de la subjetividad. Rialp, Madrid, 1967.

24 «Perciò deve star saldo che la coscienza non può partire che de se stessa, come percezione la quale (oltre l'oggetto, qualumque esso sia) include, afferma e ripete se stessa (...). La coscienza nel suo significato etimologico (...) è l'avvertenza della presenza di qualcosa ad un soggeto conoscente sia che si tratti di atti e stadi d'animo propio del soggetto stesso, come di obggeti e fatti del mondo esterno... (C. Fabro, L'Anima, Studium, Roma, 1953, p. 19).

25 «L'introspezione si distinge pertanto della consapevolezza immediata o coscienza concomitante che di necessità acompagna ogni atto psichico cosciente ch'è detto tale precisamente per l'avvertenza che l'io ha de essere in atto de sentire, volere, pensare ecc.: qui si avverte unicamente come soggetto in atto rispetto ad un oggeto. (Ibid., p. 26).

26 Ibidem. 
estrictamente refleja (in actu signato), que implica junto a lo conocido directamente por el acto cognoscitivo, la presencia de la misma subjetividad cognoscente. ${ }^{27}$ Dicha co-presencia aparece en la estructura de un único acto, en el cual, la subjetividad se expande en dos planos, haciéndose cargo del objeto (plano objetivo-temático) y de sí misma (plano concomitante o connotado).$^{28}$ De este modo, el acto cognoscitivo intelectual se manifiesta constituido por dos planos o dimensiones; uno temático, sobre el que versa el acto de modo directo, y otro, que lo acompaña siempre, donde se capta el entender que entendemos (cocemos el acto de conocer) y se aprecia a la misma subjetividad como conciendo y siendo. ${ }^{29}$

Tal vez el texto de Tomás de Aquino más completo a este respecto es el corpus del a. 8 de la q. 10 De Veritate. En el artículo el Aquinate aborda el problema del conocimiento que tiene el alma de sí misma. En el desarrollo de la cuestión distingue Santo Tomás un doble conocimiento del alma: uno por el que el alma se conoce a sí misma sólo en cuanto a lo que le es propio, otro por el que el alma conoce lo que es común a todas las almas. El primero es un conocimiento particular (de la persona) y existencial (se percibe el alma a sí misma como siendo); el segundo es el conocimiento de la naturaleza del alma (conocimiento de la esencia del alma). ${ }^{30}$

García López ${ }^{31}$ llama al primero conocimiento existencial del yo, porque es particular y se refiere a la existencia del mismo yo; al segundo lo denomina, por el contrario, conocimiento esencial ya que implica el conocimiento de la naturaleza del alma, y es de carácter universal. ${ }^{32}$

El conocimiento existencial se da en acto cuando el alma actúa. ${ }^{33}$ Este conocimiento del yo se caracteriza porque se presenta en un único acto, como dimensión consciente del mismo, ${ }^{34}$ y porque implica siempre un entender algo distinto del yo de modo directo, ya que la conciencia del yo es simplemente concomitante. ${ }^{35}$ En el conocimiento existencial del yo hay, por tanto, una dimensión objetiva que corresponde a lo que se conoce in rectoy una dimensión subjetiva, que se refiere al yo conocido in obliquo. A la dimensión objetiva le corresponde el objeto, lo conocido de modo explícito o temático; a la subjetiva le corresponde lo conocido de modo indirecto, implícito o atemático: es lo que llamaremos dimensión in-objetiva del acto cognoscitivo. ${ }^{36}$

27 «ll movimento della coscienza ne suo ritorno a se stessa: questo ritorno costituisce il terzo e più alto movimento del suo agire, dopo i due movimenti concomitanti della percezione del mondo esterno con l'apprensione dei contenuti intellettivi immeditati e della percezione concomitante del propio io» (Ibid., p. 27).

28 Millán Puelles, A., O.C., pp. 135-137.

29 Fabro, C., O.C., pp.. 20-21.

30 De Ver., q. 10, a. 8.

31 García López, J., Estudios de metafísica tomista, Eunsa, Pamplona, 1976, cap.: «El conocimiento del yo», pp. 221-251. Ibid., «La conciencia concomitante en Santo Tomás en AA. VV., «Razón y libertad. Homenaje a Antonio Millán-Puelles», Rialp, Madrid, 1990.

32 Canals Vidal, F. Sobre la esencia del conocimiento . PUB, Barcelona, 1987.

33 De Ver., q. 10, a. 8.

34 In I Sent., d. I, q. 2, a. 1, ad 2; In I Sent., d. X, q. 1, a. 5 , ad 2.

35 De Ver., q. 10, a. 8.

36 García López, O. C., p. 234. 
En cuanto al conocimiento esencial del alma, Santo Tomás lo muestra posible gracias a una reflexión por la que el alma se tematiza a sí misma y asus actos, constituyéndose en objeto de conocimiento directo.

Cuando Fabro caracteriza a la conciencia concomitante, que implica una capacidad de reflexión perfecta en la medida que es intelección de intelección, la sitúa en el ámbito del intelecto; ${ }^{37}$ ámbito en el que también incluía Santo Tomás al primum cognitum. Esta coincidencia abre una puerta para la consideración del primum cognitum que ahota trataremos de traspasar.

Sin embargo no acaban aquí todos los elementos del acto de intelección. Junto a la dimensión objetiva o plano temático, hemos identificado una dimensión inobjetiva, que es intelección de intelección; la cual implica la presencia connotada del yo cognoscente. Pero en los actos de trascendencia intencional, por los que el sujeto se trasciende a sí mismo alcanzando cognoscitivamente (intencionalmente) la realidad, la intelección de intelección también manifiesta este trascenderse hacia la realidad. Es decir, del mismo modo que se distingue la conciencia concomitante (in actu exercito) que se presenta en todo acto intelectual, de la conciencia refleja (in actu signato), en la que la subjetividad se tematiza y objetiva, volviendo sobre sí misma; del mismo modo también hay que afirmar que en todo acto cognoscitivo se da una trascendencia intencional conocida, que es condición indispensable para la presencia temática del objeto, y de la cual sabemos concomitantemente.

Este último elemento que hemos determinado, el conocomiento de la trascendencia intencional del acto cognoscitivo, está especialmente presente en el juicio, donde de modo patente el sujeto conoce su adecuarse con la realidad. Dicho conocer su adecuación se hace mediante una cierta reflexión concomitante que no implica otro acto de conocimiento, sino que se da como elemento constitutivo del mismo acto judicativo.

La reflexión veritativa, señala Cardona,$^{38}$ no consiste en ir más allá de las ideas, salir fuera del conocimiento, lo cual es absurdo, sino que se trata más bien del referirse de tal idea a la realidad. ${ }^{39}$ Esta reflexión tiene algunas semejanzas con la que se establece para el conocimiento del singular mediante la conversio ad phantasmata, ${ }^{40}$ que es considerada por Fabro, al comentarla, como reflexión in actu exercito para distinguirla de la reflexión in actu signato. ${ }^{41}$ Tanto en la reflexión veritativa como en la del conocimiento singular, la reflexión no consiste en un acto distinto, sino que se da unida al mismo acto cognoscitivo, como un elemento mismo. ${ }^{42}$

En particular, en la reflexión veritativa, el intelecto reflexiona sobre su acto y conoce tal acto y la proporción del mismo a la cosa, lo cual implica conocer también la naturaleza del principio activo, que es el intelecto, y que a ésta corresponde el conformarse con la cosa. ${ }^{43}$

37 Fabro, C., L'Anima, pp. 20-21.

38 Cardona, Metafisica de la opción intelectual. Rialp, Madrid, 1973.

39 Ibid., pp. 42-43.

40 De Ver., q. 10, a. 5: Ibid., q. 2, a. 6

41 Fabro, C., Percepción y pensamiento. Eunsa, Pamplona, 1978, pp. 328-329.

42 Cardona, $O, C .$, p. 40.

43. De Ver., q. 1, a. 9. 
Esto significa que en los actos originarios de conocimiento (conocimiento objetivo), lo que se hace presente obiective es sólo lo que -en el lenguaje de la reflexión- se llama objeto y, tan sólo de modo consectario, el conocimiento del acto de la trascendencia intencional (conocimiento del acto cognoscitivo y de su adecuarse con la realidad) y de la subjetividad que lo reliza (principio de tal acto). Sólo en la reflexión in actu signato estos tres elementos del acto cognoscitivo -el objeto en cuanto elemento de la dimensión objetiva, el acto de trascendencia intencional y la subjetividad connotada, como principios de tal acto- se tematizan y hacen presentes. ${ }^{44}$

\section{EL PRIMUM COGNITUM COMO HÁBITO INTELECTUAL}

Al intellectus, como hábito de los primeros principios, le corresponde el conocimiento del principio de no contradicción y del primum cognitum, ambos conocimiemtos indispensables para cualquier acto intelectual. Sin embargo también parece que a esta misma función cognoscitiva le correspondan las dimensiones concomitantes de la intelección (conocimiemto del acto cognoscitivo, conocimiemto del principio activo próximo, de la subjetividad que conoce, conocimiento de la trascendencia intencional).

No podemos, por otra parte, olvidar que para el conocimiento concomitante de estos elementos inobjeticos del conocimiento es también necesario el conocimiemto previo del primum cognitum sin el cual no son posibles: el conocimiento de la subjetividad, porque se conoce como ente; el conocimiento de la intencionalidad (reflexión veritativa) porque si no sabemos de la realidad en cuanto realidad, es imposible saber de la intencionalidad y sin ella, no sabemos intelectualmente que conocemos. ${ }^{45}$

Está claro que para una subjetividaad que se autotrasciende refiriéndose a alguna determinada realidad, sea necesaria -como condición de posibilidad para ese acto de trascendencia intencional- la apprehensio entis. El objeto de las viviencias originariamente cognoscitivas requiere el primum cognitum. ${ }^{46}$

Si el primum cognitum se manifiesta pues como condición de posibilidad del acto intelectual tanto por la línea de lo objetivo o temático, como por la línea de las dimensiones concomitantes o inobjetivas, podría corresponder al ens, después de haber sido aprehendido por primera vez, un estatuto inobjetivo y concomitante. ${ }^{47}$

44 Millán-Puelles, O. C., p. 181 En concreto, el texto tomista: «nullus potest cogitare se non esse cum assensu: in hoc enim quod cogitat aliquid percipit se esse» (De Ver., q. 10, a. 12, ad 7) lo interpreta L. Polo como un conocimiento habitual «Lo intelectual y lo inteligible» en Anuario Filosófico, XV-2 (1982), pp. 103-132.

45 S. Th., I, q. 16, a. 4, ad 2.

46 Millán-Puelles, A., O. C., pp. 151-161.

47 El conocimiento inobjetico en acto de la intelección de intelección por el que el alma se conoce a sí misma existencialmente podrá no requerir especie impresa; pero en el caso del ens, su presencia inobjetiva remite a otro acto originario (primera operación), el cual sí exige especie impresa y expresa. 
En cualquier acto intelectual centramos la atención sobre la determinada formalidad que consideramos temáticamente, pero a ésta la sabemos (de modo concomitante y no objetivo) como real (ens), como sabemos de la misma subjetividad como un ente. El ente es captado en toda intelección, pero no por un acto anterior a dicha intelección, sino como un elemento connotado presente en el mismo acto cognoscitivo.

¿Cómo sería esto posible? Gracias a que el intellectus es el hábito de los primeros principios, con lo cual, después de la primera captación directa o temática del ens, este puede ser conocido sin necesidad de un nuevo acto explícito o directo sobre él. ${ }^{48}$

En el libro V de la Metafisican, Aristóteles da una definición general de hábito, ${ }^{49}$ donde lo considera como algo intermedio entre el sujeto que tiene y lo tenido. El hábito es un acto que consiste en una cierta formalización de la facultad, que ésta tiene como propia y adquiere mediante una operación (no es por naturaleza), y que le abre nuevas posibilidades, se van modificando -crecen- con las operaciones posteriores..$^{50}$

El hábito de los primeros principios se diferencia del de ciencia y de la sabiduría, además de por su hábito de objetos, porque éste se da en el inicio; es más, significa el inicio de la actividad del pensamiento. ${ }^{51}$ En la prosecución de tal actividad, este hábito tiene un papel que se puede denominar activo, en cuanto el intelecto, a partir de estos primeros inteligidos, conoce todo lo demás, ya sea porque es utilizado en la misma abstracción, como porque por la vía del juicio, el intelecto -resolviendo- analiza lo hallado a la luz de este principio. ${ }^{52}$ Santo Tomás los llega a llamar ciertos instrumentos del intelecto agente por los cuales se puede hacer algo inteligible en acto. ${ }^{53}$ Efectivamente, todo objeto intelectual es regido por el principio de no contradicción; si no, no sería inteligible, ya que lo contradictorio no se puede pensar.

De este modo el ens como primum cognitum es un hábito que aparece en un primer acto de la mente por el que aferra y entra en contacto con la realidad..$^{54} \mathrm{Gracias}$ al mismo se prosigue en el conocimiento posterior, donde está presente de modo actual y concomitante, en todo acto de conocimiento intelectual. Sobre este ens puede la inteligencia reflexionar y tematizarlo, haciéndolo objeto de consideración directa. Así se explica, señala Melendo, la relación entre el primum cognitum y el ens objeto de la metafísica. ${ }^{55}$

Con nuestra relación hemos pretendido mostrar cómo desde las indicaciones de Tomás de Aquino cabe una consideración del primum cognitum según la cual éste es visto como un elemento de todo acto intelectivo, en el que está presente de modo inobjetivo y concomitante, comportándose como un principio noético.

48 Polo, L., Curso de Teoria del Conocimiento. Eunsa, Pamplona, T. II, pp. 306-309; T. III, pp. 1-27. J. Cruz Cruz, Intelecto y Razón. Eunsa, Pamplona, 1982, pp.82.

49 Met., V, 20, 1022 b 4-8.

50 Met., V, 20, 1022 b 5.

51 De Ver., q. 11, a. 1.

52 S.Th., I, q. 79, a. 8.

53 Q.D.de An., a. 5, c.

54 Melendo, T., «La expansión perfectiva del ente en el trascendental 'pulchrum'» en Estudios Filosóficos, 98, v. XXXV (1986), p. 106.

55 Ibid., O.C., pp. 106-107. 